\section{RMD Open}

Rheumatic \&

Musculoskeletal Diseases

\title{
Risk factors for venous
}

\section{thromboembolism and atherosclerotic cardiovascular disease: do they differ in patients with rheumatoid arthritis?}

\author{
Gulsen Ozen (D) , Sofia Pedro, ${ }^{2}$ Rebecca Schumacher, ${ }^{2}$ Teresa Simon (1) , \\ Kaleb Michaud (1) 1,2
}

To cite: Ozen G, Pedro S, Schumacher R, et al. Risk factors for venous thromboembolism and atherosclerotic cardiovascular disease: do they differ in patients with rheumatoid arthritis?. RMD Open 2021;7:e001618. doi:10.1136/ rmdopen-2021-001618

- Additional supplemental material is published online only. To view, please visit the journal online (http://dx.doi.org/10. 1136/rmdopen-2021-001618).

Received 4 February 2021 Accepted 14 June 2021

Check for updates

(c) Author(s) (or their employer(s)) 2021. Re-use permitted under CC BY-NC. No commercial re-use. See rights and permissions. Published by BMJ.

${ }^{1}$ Internal Medicine, University of Nebraska Medical Center, Omaha, Nebraska, USA ${ }^{2}$ FORWARD, The National Databank for Rheumatic Diseases, Wichita, Kansas, USA ${ }^{3}$ Physicians Research Center, LLC, Toms River, New Jersey, USA

\section{Correspondence to} Dr Kaleb Michaud; kmichaud@unmc.edu

\section{ABSTRACT}

Objective Venous thromboembolism (VTE) is an increasing concern in rheumatoid arthritis (RA) with little known about risk factors. We aimed to compare risk factors for unprovoked VTE and atherosclerotic cardiovascular disease (ASCVD) in patients with RA and to assess subsequent ASCVD risk after an unprovoked VTE.

Methods People with RA participating in a US-wide Iongitudinal observational registry from 1998 to 2018 were assessed for incident unprovoked VTE (deep venous thrombosis and pulmonary emboli not associated with cancer, recent surgery, hospitalisation, fracture and pregnancy) and ASCVD (myocardial infarction and stroke) validated from hospital/death records. Risk factors for VTE and ASCVD and the risk of ASCVD after an unprovoked VTE were determined using Cox proportional hazards models. Results During median (IQR) 4 (1.5-7) years of followup in 31366 patients with RA, 539 unprovoked VTE and 1648 ASCVD events were identified. The adjusted models showed increased VTE and ASCVD risk with older age, male sex, comorbidities, prior fracture, worse disability, higher disease activity and glucocorticoids. Traditional cardiovascular disease risk factors were common in both ASCVD and VTE but only increased ASCVD risk with obesity as the exception (VTE HR (95\% Cl), 1.46 (1.13-1.87)) and ASCVD, 0.58 (0.50-0.68)). ASCVD risk doubled after an unprovoked VTE (HR (95\% Cl), 2.05 (1.43-2.95)).

Conclusion Our findings suggest that unprovoked VTE is mediated by inflammation of RA and may be considered a spectrum of pan-cardiovascular syndrome.

\section{INTRODUCTION}

The risk of atherosclerotic cardiovascular disease (ASCVD) and venous thromboembolism (VTE) in patients with rheumatoid arthritis (RA) is significantly increased compared with the general population. ${ }^{12}$ In the past, it was believed that ASCVD and VTE were pathophysiologically distinct disorders. Growing data suggest that VTE is a part of cardiovascular disease (CVD) mediated by chronic inflammation. ${ }^{34}$ This is supported by

\section{Key messages}

What is already known about this subject?

- Patients with rheumatoid arthritis (RA) at increased risk of venous thromboembolism (VTE) and atherosclerotic cardiovascular disease (ASCVD), which were both shown to be significantly associated with disease activity.

- When provoking risk factors for VTE are excluded it is unknown which patients will develop VTE or ASCVD as the first cardiovascular disease (CVD) in the setting of chronic inflammation. The other CVD consequences after an unprovoked VTE in patients with RA are not clear.

What does this study add?

- This nationwide study demonstrates that traditional CVD risk factors were common both in patients with unprovoked VTE and ASCVD, but they predicted ASCVD except for obesity, which increased VTE risk.

- RA disease severity and activity parameters predicted VTE and ASCVD similarly, and hydroxychloroquine reduced both ASCVD and VTE risk. Patients with RA had a doubled risk of subsequent risk of ASCVD after an unprovoked first VTE event.

How might this impact on clinical practice or future developments?

- Unprovoked VTE may be a spectrum of pancardiovascular syndrome in patients with RA and a risk factor for a future ASCVD. Our results may help stratify the VTE and ASCVD risk and encourage physicians to integrate well-known CVD risk reduction strategies into the prevention of VTE and subsequent ASCVD.

the evidence of increased VTE risk in various chronic inflammatory disorders. ${ }^{5}$ Also, ASCVD and VTE share common mechanisms including endothelial dysfunction, blood flow changes, coagulation cascade and platelet aggregation activation, which are known to be intensified by chronic inflammation. ${ }^{67}$ \section{1}


When risk factors favouring VTE (recent surgery, hospitalisation or cancer) are excluded, it is unknown which patients will develop VTE or ASCVD as the first CVD in the setting of chronic inflammation. Except for a recent study showing an increased VTE risk with RA disease activity, well known for ASCVD, ${ }^{89}$ there is no data assessing RA or the patient characteristics for different CVDs. VTE occurs less frequently than ASCVD; however, it can cause significant mortality and morbidity. ${ }^{10}$ Recently, there is an increasing concern for VTE in patients with RA due to recent associations in the occurrence of VTE with Janus kinase inhibitors (JAKis). ${ }^{11}$ Therefore, it is important to determine the risk factors for VTE especially in comparison to ASCVD in patients with RA, to plan a mutual risk modification strategy and stratify the risk before starting a disease-modifying antirheumatic drug (DMARD), which may increase the VTE risk.

In this study, we sought to compare risk factors for unprovoked VTE and ASCVD in patients with RA as the first CVD. Then, we assessed subsequent ASCVD risk in patients with RA after an unprovoked VTE.

\section{PATIENTS AND METHODS}

We conducted a cohort study using FORWARD, The National Databank for Rheumatic Diseases, a longitudinal observational registry. Participants were recruited from US rheumatologists and were followed with selfreport semiannual questionnaires. ${ }^{12}$ Participants with RA (diagnosed by treating physician) in FORWARD from January 1998 to December 2018 who did not have any prior CVD (VTE or ASCVD) at baseline and an active cancer throughout the follow-up were included. Patients with history of hospitalisation or recent surgery were not excluded to be able to capture outcomes that require hospitalisation or surgery.

We excluded those with a "provoked" VTE, defined as a VTE following a recent ( $\leq 6$ months) hospitalisation/ surgery, fracture, cancer or pregnancy. We excluded provoked cases because these well-established VTE risk factors would skew the associations with the other patient characteristics and RA features. Also, these factors are difficult to control due to different risk magnitudes with type of surgery, anaesthesia, hospitalisation reason and duration, thromboprophylaxis method and type and stage of cancer.

For the secondary objective, among the primary cohort, patients with $\geq 1$-year participation, especially after an unprovoked VTE, were selected to allow time for an ASCVD development.

\section{Outcomes}

The outcomes were (1) incident ASCVD as composite endpoint of the first confirmed fatal or non-fatal myocardial infarction, stroke or related death and (2) incident unprovoked VTE as composite endpoint of the first confirmed deep venous thrombosis (DVT) and/or pulmonary emboli (PE) or related death. The outcome

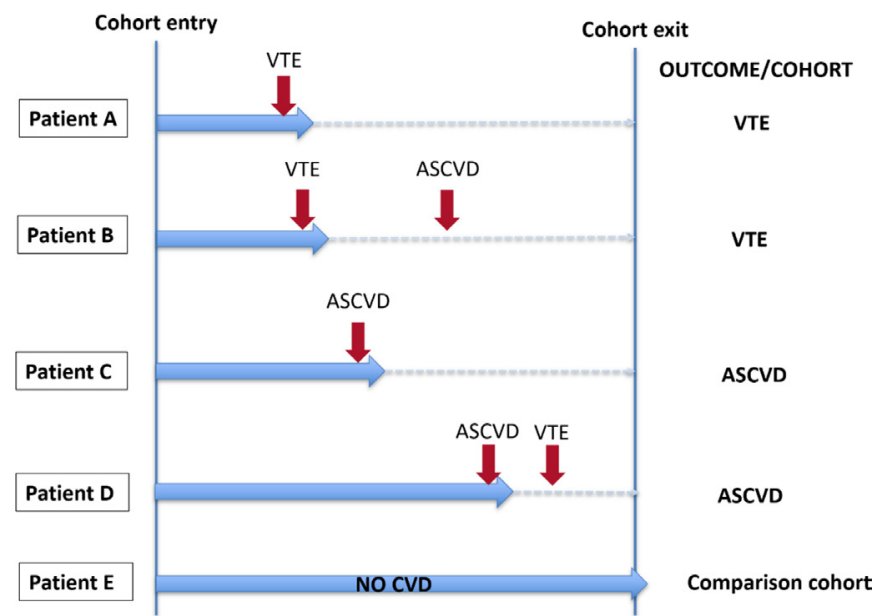

Figure 1 An example of patient allocation according to outcomes. Patients A and B-assessed for risk of VTE only; patients $\mathrm{C}$ and $\mathrm{D}$-assessed for risk of ASCVD only. Red vertical arrows indicate events, and blue horizontal arrows indicate follow-up period. ASCVD, atherosclerotic cardiovascular disease; CVD, cardiovascular disease; VTE, venous thromboembolism

for the secondary objective was incident ASCVD as described above.

All possible ASCVD and VTE events were identified from study questionnaires, hospitalisation/procedural/ death records and physician reports. If hospital/death records were not available, the patient's physician, the patient or the family was contacted with a structured, protocolised interview designed to address the reported condition. Only the confirmed events via the abovementioned process were included. Previous studies from FORWARD showed that CVD events were valid in more than $90 \%$ of cases. ${ }^{13}$ To identify and validate the CVD events, we used International Classification of Diseases, Ninth and Tenth Revision (online supplemental table 1S). Haemorrhagic cerebrovascular events or transient ischaemic attacks were not included as 'stroke' due to difficulty in confirming the event and uncertainty of the underlying atherosclerotic process.

The first of any of these CVD events during follow-up was assessed. The risk of VTE and ASCVD was analysed separately against patients without any of these outcomes. If a patient developed both events in different times, that patient could only contribute to the first occurred outcome (figure 1). This approach was chosen to avoid including overlapping patients in risk factors assessment for different events. Furthermore, development of an ASCVD with or without hospitalisation can trigger VTE or vice versa in the early period.

\section{Covariables}

Variables related to ASCVD and VTE development were collected semiannually by study questionnaires. These included age; sex; ethnicity; location of residence (rural vs urban) ${ }^{14}$; annual income; insurance type (Medicare vs others); body mass index (BMI) in WHO categories 
(normal weight reference); smoking (ever vs never); Rheumatic Disease Comorbidity Index (RDCI) excluding diabetes and hypertension as RDCI includes both ${ }^{15}$; hypertension; diabetes; exercise; pulmonary disease; chronic kidney disease (CKD); prior fracture ( $>1$ year from the outcomes); prior cancer (diagnosis before cohort entry, in remission); recent hospitalisation or surgery (within the last 6 months); RA duration; Health Assessment Questionnaire (HAQ); pain and patient global scores (0-10); DMARDs; glucocorticoids; number of previous conventional synthetic (cs), biological (b) or targeted synthetic (ts) DMARDs; and medications influencing outcomes (statins, aspirin, non-steroidal antiinflammatory drugs (NSAIDs) and oestrogen-containing or oestrogen-mimicking medications). DMARDs were assessed individually according to priorly noted associations with $\mathrm{CVD}^{16}$ : methotrexate, hydroxychloroquine (HCQ), tumour necrosis factor- $\alpha$ inhibitors (TNFis) and other b/tsDMARDs. As the number of patients on nonTNFi bDMARDs and JAKis was limited, they were not assessed individually.

For RA disease activity, we used Patient Activity Scale (PAS), a patient-reported composite disease activity scale calculated by using HAQ and pain and patient global scores. ${ }^{17}$ We categorised disease activity as remission/low disease activity (PAS $\leq 3.70$ ) and moderate/high disease activity (PAS $>3.70)$.

\section{Statistical analysis}

Baseline characteristics of patients with RA by the outcomes were summarised by using descriptive statistics. ASCVD and VTE incidence rates were calculated by dividing the number of events per 1000 patient-years of follow-up with $95 \%$ CI.

We constructed different multivariable Cox proportional hazards models to assess the risk factors for ASCVD and VTE using the covariables above. To assess the disease activity for the risk of outcomes, we created a different model by adding the PAS variable (remission/ low disease activity vs moderate/high disease activity) and removing individual components of PAS (HAQ and pain and patient global scores). The study follow-up started at the cohort entry and continued until the participant developed an outcome or was censored at death, loss to follow-up or end of study period.

For the secondary objective, another Cox proportional hazards model was created to assess the risk of ASCVD in patients with unprovoked VTE. The same covariables for the main analysis were used in this model. For this part of the analysis, the study follow-up started at the time of the VTE diagnosis and continued until the participant developed an ASCVD or was censored at death, loss to follow-up or end of study period.

All assessed covariables including medication exposures were time varying indicating current use. Missing covariables were replaced by using multiple imputations by chained equations to create multiple imputed datasets for analyses (annual income had 6\% missing; all other variables had $1 \%$ missing).${ }^{18}$ All tests were two sided and considered statistically significant when $p<0.05$. All statistical analyses were performed using Stata V.15.0.

\section{RESULTS}

A total of 31366 patients with RA were assessed for VTE and ASCVD risk. From a total of 37300 patients with RA, 5934 patients were excluded (prior VTE or any provoked VTE $=276 / 320$, prior $\mathrm{ASCVD}=2012$ and active cancer=3326). We identified 539 first unprovoked VTE and 1648 first ASCVD events during median (IQR) 4 (1.5-7) years of follow-up (supplementary results). Baseline characteristics of patients who did or did not develop any outcome are shown in table 1. Patients who developed ASCVD were older and less likely to be a woman than patients who developed VTE and who did not develop any CVD. Patients who developed VTE had higher BMIs and more comorbidities than those who developed ASCVD or did not develop any CVD. The frequency of CVD risk factors (diabetes, hypertension, smoking and CKD), prior medical history of cancer, fracture, disability and disease activity scores were higher in patients who developed any outcome compared with who did not, but these factors did not differ among patients with VTE and ASCVD. Glucocorticoid, non-TNFi bDMARD, JAKi and statin use at baseline were more frequent in patients who developed VTE than patients who developed ASCVD or did not develop any CVD.

The incidence rate of ASCVD was approximately five times higher than unprovoked VTE in patients with RA, 1.57 (95\% CI 1.51 to 1.63 ) versus 0.34 (95\% CI 0.31 to 0.37 ) per 1000 patients-years, respectively. Multivariable adjusted models showed that older age, male sex, higher comorbidity and HAQ scores, prior fracture and moderate/high disease activity were associated with both increased VTE and ASCVD risks (table 2 and online supplemental table 3S). Age and sex were stronger risk factors for ASCVD than for VTE, whereas prior fracture was a stronger risk factor for VTE than for ASCVD. Among the traditional CVD risk factors, diabetes, hypertension and CKD were associated with increased ASCVD risk only. Obesity (BMI $\geq 30 \mathrm{~kg} / \mathrm{m}^{2}$ ) was associated with decreased ASCVD risk (HR $(95 \% \mathrm{CI}), 0.56(0.48-0.66))$ and increased VTE risk (HR (95\% CI), 1.47 (1.14-1.89)). Rather, being underweight $\left(\mathrm{BMI}<18.5 \mathrm{~kg} / \mathrm{m}^{2}\right)$ was associated with increased ASCVD risk (HR (95\% CI), 1.53 (1.12-2.07)) with a non-significant decrease in VTE risk (HR (95\% CI), 0.66 (0.30-1.61)).

Regarding medications, HCQ use was associated with $\sim 20 \%$ risk reduction of ASCVD and VTE (table 2). Glucocorticoids were associated with increased ASCVD and VTE, with a slightly higher risk for VTE (HR (95\% CI), 1.37 (1.22 to 1.56 ) vs 1.99 (1.66 to 2.40$]$ ). No ASCVD or VTE risk changes were noted with other DMARDs, statins or NSAIDs.

For the secondary objective, we excluded patients with <1 year of follow-up. Among 25576 patients, we identified 1283 ASCVD events in $25070(5.1 \%)$ patients 
Table 1 Baseline characteristics of patients with RA who did not develop any CVD or who developed a VTE or an ASCVD as the first $C V D^{*}$

\begin{tabular}{|c|c|c|c|c|}
\hline Variables & $\begin{array}{l}\text { No VTE or ASCVD, } \\
n=29179\end{array}$ & $\begin{array}{l}\text { VTE as first CVD, } \\
n=539\end{array}$ & $\begin{array}{l}\text { ASCVD as first CVD, } \\
n=1648\end{array}$ & $\begin{array}{l}\text { P value for VTE } \\
\text { vs ASCVD }\end{array}$ \\
\hline Age, years & $58.9(13.6) \dagger$ & $66.5(12.0)$ & $71.8(10.3)$ & $<0.001$ \\
\hline Woman, \% & $82.2 \ddagger$ & 79.4 & 71.4 & $<0.001$ \\
\hline Caucasian, \% & $94.6 \neq$ & 93.5 & 97.5 & $<0.001$ \\
\hline RA duration, years & $15.4(11.1) \dagger$ & $19.6(13.0)$ & $19.4(13.5)$ & 0.843 \\
\hline $\mathrm{BMI}, \mathrm{kg} / \mathrm{m}^{2}$ & $28.5(6.4) \dagger$ & $30.4(7.8)$ & $27.0(5.6)$ & $<0.001$ \\
\hline Obesity, \% & $28.8 \dagger$ & 40.6 & 24.0 & $<0.001$ \\
\hline Exercise, \% & $9.4 \dagger$ & 8.0 & 14.1 & 0.002 \\
\hline $\mathrm{RDCl}(0-9)$ & $1.8(0.6) \dagger$ & $2.7(1.8)$ & $2.2(1.6)$ & $<0.001$ \\
\hline Ever smoked, \% & $40.8 \ddagger$ & 43.4 & 46.5 & 0.206 \\
\hline Diabetes, \% & $9.8 \dagger$ & 17.4 & 15.3 & 0.235 \\
\hline Hypertension, \% & $28.5 \dagger$ & 49.4 & 47.3 & 0.432 \\
\hline Pulmonary disease, \% & $6.0 \dagger$ & $13.4 \S$ & 11.8 & 0.356 \\
\hline Chronic kidney disease, \% & 7.4 & 12.1 & 14.5 & 0.189 \\
\hline Prior fracture, \%ף & $2.4 \dagger$ & 10.1 & 7.7 & 0.189 \\
\hline Prior cancer, \%ף & $7.6 \dagger$ & 15.7 & 15.0 & 0.737 \\
\hline Recent hospitalisation, \%* & $7.3 \dagger$ & 8.1 & 10.1 & 0.001 \\
\hline Recent surgeries, $\%^{* *}$ & 3.1 & 4.1 & 5.9 & 0.004 \\
\hline HAQ disability (0-3) & $1.1(0.7) \dagger$ & $1.4(0.7)$ & $1.4(0.8)$ & 0.661 \\
\hline PAS (0-10) & $4.0(2.3) \dagger$ & $4.6(2.1)$ & $4.5(2.3)$ & 0.697 \\
\hline Glucocorticoid current, \% & $33.7 \dagger$ & 50.9 & 46.2 & 0.063 \\
\hline Glucocorticoid ever, \% & $71.8+$ & 84.9 & 79.5 & 0.006 \\
\hline Methotrexate, \% & $47.6 \dagger$ & 49.4 & 48.5 & 0.743 \\
\hline Hydroxychloroquine, \% & $20.3+$ & 17.9 & 15.1 & 0.123 \\
\hline TNFi, \% & $30.2 \dagger$ & 36.5 & 33.2 & 0.161 \\
\hline Other b/tsDMARDs, \% & $8.9+$ & 11.9 & 3.9 & $<0.001$ \\
\hline NSAID, \% & $49.0 \dagger$ & 38.2 & 43.8 & 0.023 \\
\hline Aspirin, \% & $14.3+$ & 22.4 & 26.9 & 0.038 \\
\hline Statin, \% & $12.9 \dagger$ & 26.9 & 20.0 & 0.001 \\
\hline Oestrogen, \% & $15.9 \dagger$ & 11.5 & 11.9 & 0.878 \\
\hline
\end{tabular}

*The values are mean (SD) unless indicated otherwise.

$\dagger \mathrm{P}<0.01$ for no CVD versus VTE or ASCVD.

$\ddagger P<0.01$ for no CVD versus ASCVD.

$\S$ Pulmonary disease excluding pulmonary emboli.

IPrior fracture and cancer diagnosis $>1$ year before cohort entry and not on active treatment.

${ }^{*}$ Recent hospitalisation or surgery within the last 6 months.

ASCVD, atherosclerotic cardiovascular disease; BMI, body mass index; b/tsDMARDs, biological or targeted synthetic disease-modifying antirheumatic drugs; CVD, cardiovascular disease; HAQ, Health Assessment Questionnaire; NSAID, non-steroidal anti-inflammatory drug; PAS, Patient Activity Scale; RA, rheumatoid arthritis; RDCI, Rheumatic Diseases Comorbidity Index; TNFi, tumour necrosis factor- $\alpha$ inhibitor; VTE, venous thromboembolism.

without baseline CVD and 38 in $506(7.5 \%)$ patients with an unprovoked VTE during median (IQR) 4.7 (2.4-8.9) years of follow-up. Patients with an unprovoked VTE had worse RA characteristics (disease duration, HAQ, disease activity and glucocorticoid use) and more frequent traditional CVD risk factors (diabetes, hypertension and obesity) than patients without CVD at baseline (online supplemental table $2 \mathrm{~S}$ ). The crude incidence rate of
ASCVD in patients with VTE was approximately threefold higher than patients without CVD at baseline (table 3). After adjusting for covariables, we found that ASCVD risk was significantly increased in patients with an unprovoked VTE (HR (95\% CI), 2.05 (1.43 to 2.95$)$ ). The risk increase was higher in patients with PE than those with DVT only (HR (95\% CI), 2.52 (1.57 to 4.04) vs 1.93 (1.22 to 3.06)) (table 3). 
Table 2 Multivariable associations between RA disease and patient characteristics with VTE and ASCVD risk*

\begin{tabular}{|c|c|c|}
\hline Variables & $\begin{array}{l}\text { aHR }(95 \% \mathrm{Cl}) \text { for } \\
\text { VTE versus no } \\
\text { CVD }\end{array}$ & $\begin{array}{l}\text { aHR }(95 \% \mathrm{Cl}) \text { for } \\
\text { ASCVD versus no } \\
\text { CVD }\end{array}$ \\
\hline \multicolumn{3}{|l|}{ Age groups } \\
\hline$<45$ years & Reference & Reference \\
\hline $45-64$ years & 1.61 (1.04 to 2.55$)$ & $3.13(1.91$ to 4.92$)$ \\
\hline$\geq 65$ years & 2.32 (1.49 to 3.75$)$ & $7.94(4.88$ to 13.21$)$ \\
\hline Man & 1.37 (1.05 to 1.73$)$ & 1.79 (1.55 to 2.08$)$ \\
\hline Caucasian & 0.96 (0.69 to 1.42$)$ & 1.19 (0.94 to 1.44$)$ \\
\hline RA duration, years & $1.00(0.99$ to 1.01$)$ & $1.00(0.99$ to 1.00$)$ \\
\hline \multicolumn{3}{|l|}{$\mathrm{BMI}$ in WHO categories } \\
\hline Underweight & $0.66(0.30$ to 1.61$)$ & $1.53(1.12$ to 2.07$)$ \\
\hline Normal weight & Reference & Reference \\
\hline Overweight & $1.04(0.82$ to 1.41$)$ & $0.73(0.61$ to 0.82$)$ \\
\hline Obese & 1.47 (1.14 to 1.89$)$ & $0.56(0.48$ to 0.66$)$ \\
\hline Exercise & 1.03 (0.90 to 1.16$)$ & 1.15 (0.99 to 1.19$)$ \\
\hline $\mathrm{RDCl}$ & 1.20 (1.14 to 1.31$)$ & $1.13(1.08$ to 1.19$)$ \\
\hline Ever smoked & 0.91 (0.76 to 1.12$)$ & 1.07 (0.97 to 1.20$)$ \\
\hline Diabetes & 0.99 (0.76 to 1.32$)$ & 1.39 (1.20 to 1.62$)$ \\
\hline Hypertension & 1.19 (0.90 to 1.50$)$ & 1.21 (1.07 to 1.39$)$ \\
\hline Chronic kidney disease & 1.08 (0.88 to 1.45$)$ & 1.31 (1.10 to 1.66$)$ \\
\hline Pulmonary disease & 0.92 (0.67 to 1.22$)$ & 1.00 (0.99 to 1.21$)$ \\
\hline Prior fracture $†$ & $1.62(1.39$ to 1.91$)$ & $1.15(1.01$ to 1.29$)$ \\
\hline Prior cancer† & 1.10 (0.81 to 1.42$)$ & 1.05 (0.88 to 1.19$)$ \\
\hline Recent hospitalisation§ & 1.09 (0.89 to 1.67$)$ & $1.06(0.91$ to 1.23$)$ \\
\hline Recent surgeries§ & 1.18 (0.93 to 2.02$)$ & $1.14(0.99$ to 1.31$)$ \\
\hline HAQ disability (0-3) & $1.22(1.04$ to 1.44$)$ & $1.29(1.15$ to 1.41$)$ \\
\hline $\begin{array}{l}\text { Moderate/high disease } \\
\text { activity versus remission/ } \\
\text { low disease activity } \neq\end{array}$ & $1.31(1.07$ to 1.61$)$ & $1.32(1.18$ to 1.50$)$ \\
\hline \multicolumn{3}{|l|}{ Medication use } \\
\hline Glucocorticoids & 1.99 (1.66 to 2.40$)$ & 1.37 (1.22 to 1.56$)$ \\
\hline Methotrexate & 1.01 (0.84 to 1.20$)$ & 0.89 (0.77 to 1.00$)$ \\
\hline Hydroxychloroquine & 0.79 (0.62 to 0.98$)$ & $0.81(0.71$ to 0.92$)$ \\
\hline TNFi & 1.06 (0.82 to 1.30$)$ & $1.00(0.84$ to 1.15$)$ \\
\hline Other b/tsDMARDs & 0.85 (0.51 to 1.16$)$ & $1.08(0.78$ to 1.50$)$ \\
\hline NSAID & 0.79 (0.65 to 1.04$)$ & $0.96(0.90$ to 1.01$)$ \\
\hline Aspirin & 0.81 (0.64 to 1.02$)$ & 1.09 (0.98 to 1.32$)$ \\
\hline Statin & 1.01 (0.79 to 1.28$)$ & 1.10 (0.95 to 1.28$)$ \\
\hline Oestrogen & 0.94 (0.71 to 1.27$)$ & 0.89 (0.73 to 1.04$)$ \\
\hline
\end{tabular}

*The model also included location of residence, insurance type, annual income and calendar year.

†Prior fracture and cancer diagnosis $>1$ year before cohort entry and not on active treatment.

$\ddagger$ Disease activity assessed by Patient Activity Scale (PAS) (evaluated in a separate model without individual components of PAS). $\S$ Recent hospitalisation or surgery within the last 6 months. aHR, adjusted HR; ASCVD, atherosclerotic cardiovascular disease; $\mathrm{BMI}$, body mass index; b/tsDMARDs, biological or targeted synthetic disease-modifying antirheumatic drugs; CVD, cardiovascular disease; $H A Q$, Health Assessment Questionnaire; NSAID, non-steroidal anti-inflammatory drug; RA, rheumatoid arthritis; RDCl, Rheumatic Diseases Comorbidity Index; TNFi, tumour necrosis factor- $\alpha$ inhibitor; VTE, venous thromboembolism.

\section{DISCUSSION}

In this large cohort study, the first to investigate the differential risk factors for first unprovoked VTE versus ASCVD in patients with RA, we found that VTE and ASCVD share common risk factors including RA disease activity and severity parameters. The main risk difference for VTE over ASCVD was traditional CVD risk factors except for obesity, which favoured VTE development. Furthermore, we found that patients with RA with an unprovoked VTE had twofold increased risk of developing ASCVD.

VTE used to be considered a condition mainly triggered by a hospitalisation, major surgery, trauma, immobilisation or cancer. However, it was noted that $\sim 30 \%-50 \%$ of cases have no provoking factors. ${ }^{19}$ The general population studies suggested that unprovoked VTE is linked to both inflammation and ASCVD. ${ }^{20-22}$

Previous RA studies mainly focused on increased VTE risk compared with the general population ${ }^{23-25}$ or with certain DMARDs like JAKis. ${ }^{11} 2627$ Few studies in RA showed an increased VTE risk with obesity, recent arthroplasty or hospitalisation, exposure to aspirin, bDMARDs and csDMARDs other than methotrexate and $\mathrm{HCQ}^{23}$ severe extra-articular RA manifestations ${ }^{28}$ and other comorbidities. ${ }^{29}$ Regarding disease duration, the VTE risk was either highest at the first year ${ }^{29}$ or steadily elevated throughout the disease. ${ }^{24}$ The only study assessing disease activity on VTE risk demonstrated that VTE risk linearly increased with increasing disease activity. ${ }^{8}$ Traditional CVD risk factors for VTE risk were evaluated in a study (pooled analysis of all immune-mediated diseases), which showed obesity and current smoking along with history of a fracture or cancer, glucocorticoids and oral contraceptives increased VTE risk. ${ }^{5}$

It's important to note that these studies included both provoked and unprovoked VTE events. Although some adjusted their analyses for recent hospitalisations, major surgeries or cancer, it is hard to determine how much of the risk can be attributable to these heterogenous provoking factors versus other factors. VTE risk varies with the type of surgery, anaesthesia, hospitalisation reason and duration, thromboprophylaxis method and cancer type /stage. ${ }^{30}$ Lastly, above-mentioned studies included patients with prior VTE or ASCVD, and only some adjusted for this important recurrence risk in the models.

Our study is the first to assess the risk factors for unprovoked VTE events compared with ASCVD in the setting of chronic inflammation, in RA. We found that male sex, older age, other comorbidities, prior fracture, HAQ disability, moderate/high disease activity and glucocorticoids were associated with increased risk of first unprovoked VTE and ASCVD with small differences in risk magnitudes. Interestingly, the frequency of traditional CVD risk factors except for obesity was not different in patients who developed VTE compared with those who developed ASCVD. However, these factors reached statistical significance only for ASCVD risk. The general population studies assessing traditional CVD risk factors 
Table 3 Crude incidence rates and risk of ASCVD in patients with RA by unprovoked VTE

\begin{tabular}{|c|c|c|c|c|c|c|c|}
\hline & $\begin{array}{l}\text { Events/ } \\
\text { patients }\end{array}$ & $\%$ & $\begin{array}{l}\text { Patient- } \\
\text { years }\end{array}$ & $\begin{array}{l}\text { Incidence rate } \\
(95 \% \mathrm{CI})^{\star}\end{array}$ & $\begin{array}{l}\text { Unadjusted } \\
\text { HR }(95 \% \mathrm{Cl})\end{array}$ & $\begin{array}{l}\text { Adjusted HR for age, } \\
\text { sex and year }(95 \% \mathrm{Cl})\end{array}$ & $\begin{array}{l}\text { Adjusted HR } \\
(95 \% \mathrm{Cl}) \dagger\end{array}$ \\
\hline $\begin{array}{l}\text { Patients without } \\
\text { prior VTE or } \\
\text { ASCVD }\end{array}$ & $1283 / 25070$ & 5.1 & 147735 & $\begin{array}{l}8.68 \\
(8.22 \text { to } 9.17)\end{array}$ & - & - & - \\
\hline $\begin{array}{l}\text { Patients with first } \\
\text { unprovoked VTE } \\
\text { (no prior ASCVD } \\
\text { or VTE) }\end{array}$ & $38 / 506$ & 7.5 & 16152 & $\begin{array}{l}23.53 \\
(17.12 \text { to } 32.33)\end{array}$ & $\begin{array}{l}2.50 \\
(1.81 \text { to } 3.44)\end{array}$ & $\begin{array}{l}2.32 \\
(1.69 \text { to } 3.11)\end{array}$ & $\begin{array}{l}2.05 \\
(1.43 \text { to } 2.95)\end{array}$ \\
\hline $\begin{array}{l}\text { Deep venous } \\
\text { thrombosis }\end{array}$ & $21 / 325$ & 6.5 & 11150 & $\begin{array}{l}18.83 \\
\text { (12.28 to } 28.87)\end{array}$ & $\begin{array}{l}2.03 \\
(1.32 \text { to } 3.12)\end{array}$ & $\begin{array}{l}2.00 \\
\text { (1.39 to } 3.09)\end{array}$ & $\begin{array}{l}1.93 \\
\text { (1.22 to } 3.06)\end{array}$ \\
\hline $\begin{array}{l}\text { Pulmonary } \\
\text { emboli }\end{array}$ & $17 / 181$ & 9.4 & 5002 & $\begin{array}{l}33.99 \\
(21.13 \text { to } 54.67)\end{array}$ & $\begin{array}{l}3.78 \\
(2.50 \text { to } 5.70)\end{array}$ & $\begin{array}{l}2.98 \\
\text { (1.92 to } 4.89)\end{array}$ & $\begin{array}{l}2.52 \\
\text { (1.57 to } 4.04)\end{array}$ \\
\hline
\end{tabular}

*Per 1000 patient-years.

†Adjusted for age; sex; disease duration; socioeconomic status (annual income, insurance and location of residency); ethnicity; smoking; hypertension; diabetes; chronic kidney disease; comorbidity index; body mass index; Health Assessment Questionnaire; patient global and pain scores; glucocorticoid use disease-modifying antirheumatic drugs (DMARDs) including methotrexate, hydroxychloroquine, tumour necrosis factor- $\alpha$ inhibitor (TNFi) and non-TNFi biological DMARDs (bDMARDs); non-steroidal antiinflammatory drugs; statins; fracture; pulmonary disease; prior count of conventional synthetic DMARDs (csDMARDs) and bDMARDs; and calendar year.

ASCVD, atherosclerotic cardiovascular disease; RA, rheumatoid arthritis; VTE, venous thromboembolism.

for VTE only found older age, male sex and obesity consistently associated with increased unprovoked VTE risk. $^{31-33}$

We also found that obesity was associated with increased VTE risk but a decreased ASCVD risk in patients with RA. This discordance is intriguing as the disease activity, severity and disability measures were associated with VTE and ASCVD similarly. Previous RA studies have suggested that obesity is associated with lower ASCVD and mortality ${ }^{13} 34$ despite increasing CVD risk factors like diabetes. ${ }^{35}$ It is unclear if this paradoxical effect is accurate or the result of incomplete capture of RA severity/ inflammatory measures or indicative of inadequacy of BMI to reflect adiposity in RA. Although obesity and VTE association can partly be explained with increased immobility in RA, it is unknown if obesity has different effects on the venous vasculature through vicinity of fat tissue or activation of different pathways more involved in VTE like coagulation cascade.

Despite not being a primary objective, we included medications in the analysis due to their potential riskmodifying effects. We found glucocorticoids were associated with increased risk of ASCVD and VTE. Although this is well known for ASCVD,${ }^{16}$ it has not been assessed before for unprovoked VTE in RA. HCQ was associated with 20\% risk reduction of ASCVD and VTE in patients with RA. The antithrombotic effects of HCQ are well known in patients with connective tissue disorders with or without antiphospholipid antibodies. ${ }^{36}$ In RA, HCQ was found to reduce both ASCVD and its risk factors like diabetes and dyslipidaemia. ${ }^{35}{ }^{37}$ Our findings suggest that HCQ may also reduce VTE risk in addition to ASCVD risk in patients with RA.

Another noteworthy finding of our study is increased ASCVD risk after an unprovoked VTE. This association was shown in the general population for unprovoked VTE events. ${ }^{20}{ }^{38}$ This link was partially attributed to obesity in the general population as it is the only consistent risk factor for both. However, as mentioned above, the effect of obesity on VTE and ASCVD is discordant in patients with RA. One might speculate that PE can change cardiac haemodynamics and trigger a cardiac event. However, DVT without a PE was also associated with increased ASCVD risk. Also, the majority of the ASCVD events $(88 \%)$ occurred $>90$ days after the unprovoked VTE in our study. This association has important clinical implications. Although traditional CVD risk factors do not seem to be associated with a VTE risk, patients with VTE have these risk factors, and the occurrence of VTE should be considered as an opportunity to reassess care gaps for traditional CVD risk factors.

Our study has some limitations. First, our event rates were lower than the prior studies assessing VTE and ASCVD in patients with RA. However, we excluded patients with provoked VTE events and with prior ASCVD or VTE. Although our strict validation process may have some minor effect on the event capture with a high accuracy rate, ${ }^{13}$ our findings are still valuable with internal comparison group subject to the same limitation. Second, although it was not our primary aim, we could not assess the risk of VTE with JAKi due to inadequate number of patients on these medications. Third, this observational study is also subject to survivor bias with disease duration $>15$ years. Despite that, in a subgroup analysis of patients with disease duration $<5$ years, the associations found in the main analysis remained similar with some loss of statistical significance due to smaller sample size (data not shown). Fourth, we did not have data regarding seropositivity, acute-phase reactants, hereditary thrombophilia or antiphospholipid antibodies. However, this is unlikely to alter our results as the prevalence of 
hereditary thrombophilia is low in the population and not reported to be higher in RA. Lastly, although we have data regarding many comorbidities, we did not have data about adequacy of traditional CVD risk factor control such as Alc.

In conclusion, we found evidence of common risk factors for unprovoked VTE and ASCVD in RA including worse disability, high disease activity, prior fracture, other comorbidities, glucocorticoids and not using HCQ. The main difference was traditional CVD risk factors, which mostly predicted ASCVD except for obesity, a risk factor for VTE. Patients with RA with an unprovoked VTE had a doubled risk of developing an ASCVD. Our findings support that unprovoked VTE may be a spectrum of pancardiovascular syndrome in patients with $\mathrm{RA}$ and a risk factor for a future ASCVD. Given the frequent CVD risk factors in VTE and increased subsequent ASCVD risk, clinicians should remember that inflammatory cytokines circulate throughout the entire vascular system and consider integrating well-known CVD risk reduction strategies into the prevention of VTE and subsequent ASCVD.

\section{Twitter Kaleb Michaud @Dr_K}

Contributors GO, SP, RS, TAS and KM had full access to all the data in the study and take responsibility for the integrity of the data and the accuracy of the data analysis. Study concept and design: all authors. Analysis of data: G0 and SP. Interpretation of data and drafting of the manuscript: all authors.

Funding KM receives grant support from the Rheumatology Research Foundation. Competing interests None declared.

Patient consent for publication Not required.

Ethics approval Ascension Via Christi Hospitals Wichita, Inc. Institutional Review Board IRB number: IRB00001674 and FWA number: FWA00001005. Protocol Title: The National Databank for Rheumatic Disease, Improving Long Term Outcomes in Arthritis.

Provenance and peer review Not commissioned; externally peer reviewed.

Data availability statement Data are not publicly available.

Open access This is an open access article distributed in accordance with the Creative Commons Attribution Non Commercial (CC BY-NC 4.0) license, which permits others to distribute, remix, adapt, build upon this work non-commercially, and license their derivative works on different terms, provided the original work is properly cited, appropriate credit is given, any changes made indicated, and the use is non-commercial. See: http://creativecommons.org/licenses/by-nc/4.0/.

\section{ORCID iDs}

Gulsen Ozen http://orcid.org/0000-0002-5423-393X

Teresa Simon http://orcid.org/0000-0003-4042-0715

Kaleb Michaud http://orcid.org/0000-0002-5350-3934

\section{REFERENCES}

1 Avina-Zubieta JA, Thomas J, Sadatsafavi M, et al. Risk of incident cardiovascular events in patients with rheumatoid arthritis: a metaanalysis of observational studies. Ann Rheum Dis 2012;71:1524-9.

2 Ogdie A, Kay McGill N, Shin DB, et al. Risk of venous thromboembolism in patients with psoriatic arthritis, psoriasis and rheumatoid arthritis: a general population-based cohort study. Eur Heart J 2018;39:3608-14.

3 Glynn RJ, Ridker PM. Inflammation, venous thromboembolism, and what we can do about it. Eur Heart J 2018:39:3615-7.

4 Piazza G, Goldhaber SZ. Venous thromboembolism and atherothrombosis: an integrated approach. Circulation 2010;121:2146-50.

5 Galloway J, Barrett K, Irving P, et al. Risk of venous thromboembolism in immune-mediated inflammatory diseases: a UK matched cohort study. RMD Open 2020;6:e001392.

6 Fox EA, Kahn SR. The relationship between inflammation and venous thrombosis. A systematic review of clinical studies. Thromb Haemost 2005;94:362-5.
7 Silvestri E, Scalera A, Emmi G, et al. Thrombosis in autoimmune diseases: a role for immunosuppressive treatments? Semin Thromb Hemost 2016;42:650-61.

8 Molander $\mathrm{V}$, Bower $\mathrm{H}$, Frisell $\mathrm{T}$, et al. Risk of venous thromboembolism in rheumatoid arthritis, and its association with disease activity: a nationwide cohort study from Sweden. Ann Rheum Dis 2021;80:169-75.

9 Solomon DH, Reed GW, Kremer JM, et al. Disease activity in rheumatoid arthritis and the risk of cardiovascular events. Arthritis Rheumatol 2015;67:1449-55.

10 Heit JA, Silverstein MD, Mohr DN, et al. Predictors of survival after deep vein thrombosis and pulmonary embolism: a populationbased, cohort study. Arch Intern Med 1999;159:445-53.

11 Mease P, Charles-Schoeman C, Cohen S, et al. Incidence of venous and arterial thromboembolic events reported in the tofacitinib rheumatoid arthritis, psoriasis and psoriatic arthritis development programmes and from real-world data. Ann Rheum Dis 2020;79:1400-13.

12 Michaud K. The National data bank for rheumatic diseases (NDB). Clin Exp Rheumatol 2016;34:S100-1.

13 Wolfe F, Michaud K. The risk of myocardial infarction and pharmacologic and nonpharmacologic myocardial infarction predictors in rheumatoid arthritis: a cohort and nested case-control analysis. Arthritis Rheum 2008;58:2612-21.

14 Rural-Urban Commuting area codes. Available: https://www.ers. usda.gov/data-products/rural-urban-commuting-area-codes/

15 Michaud K, Wolfe F. Comorbidities in rheumatoid arthritis. Best Pract Res Clin Rheumatol 2007;21:885-906.

16 Ozen G, Pedro S, Michaud K. The risk of cardiovascular events associated with disease-modifying antirheumatic drugs in rheumatoid arthritis. J Rheumatol 2021;48:648-55.

17 Wolfe F, Michaud K, Pincus T. A composite disease activity scale for clinical practice, observational studies, and clinical trials: the patient activity scale (PAS/PAS-II). J Rheumatol 2005;32:2410-5.

18 Rubin DB. Multiple imputation in nonresponse for surveys. New York Wiley, 1987.

19 White RH. The epidemiology of venous thromboembolism. Circulation 2003;107:41--8.

20 Prandoni P, Ghirarduzzi A, Prins MH, et al. Venous thromboembolism and the risk of subsequent symptomatic atherosclerosis. $J$ Thromb Haemost 2006;4:1891-6.

21 Spencer FA, Ginsberg JS, Chong A, et al. The relationship between unprovoked venous thromboembolism, age, and acute myocardial infarction. J Thromb Haemost 2008;6:1507-13.

22 Kunutsor SK, Seidu S, Blom AW, et al. Serum C-reactive protein increases the risk of venous thromboembolism: a prospective study and meta-analysis of published prospective evidence. Eur $J$ Epidemiol 2017;32:657-67.

23 Bacani AK, Gabriel SE, Crowson CS, et al. Noncardiac vascular disease in rheumatoid arthritis: increase in venous thromboembolic events? Arthritis Rheum 2012;64:53-61.

24 Holmqvist ME, Neovius M, Eriksson J, et al. Risk of venous thromboembolism in patients with rheumatoid arthritis and association with disease duration and hospitalization. JAMA 2012;308:1350-6.

25 Kim SC, Schneeweiss S, Liu J, et al. Risk of venous thromboembolism in patients with rheumatoid arthritis. Arthritis Care Res 2013;65:NA-7.

26 Desai RJ, Pawar A, Weinblatt ME, et al. Comparative risk of venous thromboembolism in rheumatoid arthritis patients receiving tofacitinib versus those receiving tumor necrosis factor inhibitors: an observational cohort study. Arthritis Rheumatol 2019;71:892-900.

$27 \mathrm{Kim} \mathrm{SC}$, Solomon DH, Liu J, et al. Risk of venous thromboembolism in patients with rheumatoid arthritis: initiating disease-modifying antirheumatic drugs. Am J Med 2015;128:539.e7-17.

28 Liang KP, Liang KV, Matteson EL, et al. Incidence of noncardiac vascular disease in rheumatoid arthritis and relationship to extraarticular disease manifestations. Arthritis Rheum 2006;54:642-8.

29 Chung W-S, Peng C-L, Lin C-L, et al. Rheumatoid arthritis increases the risk of deep vein thrombosis and pulmonary thromboembolism: a nationwide cohort study. Ann Rheum Dis 2014;73:1774-80.

30 Chua CC, Lim HY, Tacey M, et al. Retrospective evaluation of venous thromboembolism: are all transient provoking events the same? Eur $J$ Haematol 2017;99:18-26.

31 Ageno W, Becattini C, Brighton T, et al. Cardiovascular risk factors and venous thromboembolism: a meta-analysis. Circulation 2008;117:93-102.

32 Gregson J, Kaptoge S, Bolton T, et al. Cardiovascular risk factors associated with venous thromboembolism. JAMA Cardiol 2019;4:163-73. 
33 Mahmoodi BK, Cushman M, Anne Næss I, et al. Association of traditional cardiovascular risk factors with venous thromboembolism: an individual participant data meta-analysis of prospective studies. Circulation 2017;135:7-16.

34 Escalante A, Haas RW, del Rincón I. Paradoxical effect of body mass index on survival in rheumatoid arthritis: role of comorbidity and systemic inflammation. Arch Intern Med 2005;165:1624-9.

35 Ozen G, Pedro S, Holmqvist ME, et al. Risk of diabetes mellitus associated with disease-modifying antirheumatic drugs and statins in rheumatoid arthritis. Ann Rheum Dis 2017;76:848-54.
36 Petri M. Use of hydroxychloroquine to prevent thrombosis in systemic lupus erythematosus and in antiphospholipid antibodypositive patients. Curr Rheumatol Rep 2011;13:77-80.

37 Rempenault C, Combe B, Barnetche T, et al. Metabolic and cardiovascular benefits of hydroxychloroquine in patients with rheumatoid arthritis: a systematic review and meta-analysis. Ann Rheum Dis 2018;77:98-103.

38 Becattini C, Agnelli G, Prandoni P, et al. A prospective study on cardiovascular events after acute pulmonary embolism. Eur Heart $J$ 2005;26:77-83. 